\title{
Non-Gaussian Distributions of Returns on S\&P 500 Index
}

\author{
Sio Chong U
}

\author{
Macau University of Science and Technology \\ Avenida Wai Long, Taipa, Macau
}

\begin{abstract}
Keywords: GJR-GARCH; Fat-Tailed Distributions; Stable Paretian Distributions.
\end{abstract}
\begin{abstract}
Distributions of returns on market index are always assumed to be normal. In fact, many researchers argue that the distributions have tails fatter than normal. GARCH models illustrate that this non-normality is because of volatility clustering. This paper investigates the distribution of returns on S\&P 500 index between 2006 and 2007. It is found that the distribution is still fatter than normal even though the heteroskedasticity has been adjusted by GARCH models. Moreover, the stable GJR-GARCH model performs better than Gaussian GJR-GARCH model.
\end{abstract}

\section{Introduction}

There are lot of literatures found that the distributions of returns on financial assets are not normal $[1,2,3,4,5]$. They found that the distributions are possible asymmetric and their tails are fatter than normal. Diebold et.al. [6] used power law to describe the tail index of the distributions of returns on financial assets. Bali [7] applied the extreme value approach to show that the distribution of returns on market index had high peaks and fat tails. Based on the ARCH model in [8], Bollerslev [2] had proposed the generanlized autoregressive conditional heteroskedasticity $(\mathrm{GARCH})$ model. Bollerslev [9] showed that the fat tails of the distributions of returns on market indices are because of volatility clustering. Mandelbrot [4, 10, 11], Fama [3, 12, 13, 14] are the pioneers who applied the family of stable Paretian distributions to the returns on financial assets. McCulloch [15, 5], Nolan [16] and Nolan et.al. [17] proposed some numerical methods to estimate the parameters of stable Paretian distributions. After that, the stable Paretian distributions are widely used. Stable Paretian distribution has four parameters. Charateristic exponent $(\alpha)$ is the tail index of the distribution. If $\alpha$ is less than 2, the second or higher moments of the random variables will be infinite. In other words, the distribution may have infinite variance. However, the scale factor $(c)$ will capture the despersion of the distribution which likes the $\sigma$ in normal distributions. The skewness $(\beta)$ described the symmetricity of the distribution. The distribution has positive (negative) skewness when $\beta>0(\beta<0)$ and the distribution is symmetric when $\beta=0$. The location $(\delta)$ shows the qausi-center of the distribution which is similar to the mean in the normal distribution. Moreover, the stable Paretian distribtuion will reduce to normal when $\alpha=2$ and $\beta=0$.

This paper investigates the tail index and the skewness of the distribution of daily returns on S\&P 500 index. Engle [18] showed that GJR-GARCH model had better performance on modeling the impact of good news and bad news. According to normal GJR-GARCH model, we released the distribution in this model. We employed the stable Paretian distribution to capture the leptokurtic and the skewness of the conditional distributions in the GARCH models. We compared the goodness-of-fit between stable Paretian distribution and the normal distribution. Since the stable Paretian distributions nested the normal distributions, we can make the hypothesis testing with $\mathrm{H}_{0}: \alpha=2 ; \mathrm{H}_{\mathrm{A}}: \alpha<2$. We used the daily returns on S\&P 500 index from $1^{\text {st }}$ January 2006 to $31^{\text {st }}$ December 2007 to fit stable GJR-GARCH model and normal GJR-GARCH model. The results highly rejected the null hypothesis, and conclude that the conditional distributions of returns on S\&P index had fat tails and asymmetric.

The rest of the paper shows that methodology and the model in next section. Data and empirical results have been given in Section 3. Finally, we have made the conclusion.

\section{Methodology}

The difficulty of using stable Paretian distribution is that the density function did not have explicit 
form. Zolotarev [19] studied the properties of stable Paretian distributions, and he also described the meanings of the parameters of stable Paretian distributions. McCulloch [5] and Nolan [20] provided some numerical methods to estimate the parameters of stable Paretian distributions and the values of pdf and cdf. The characteristic function of stable random variable $($ ) has the main role on the estimation.

$$
\ln (x ; \alpha, \beta, c, \delta)= \begin{cases}\exp \left\{\mathrm{i} \delta x-|c x|^{\alpha}\left[1-\mathrm{i} \beta \operatorname{sign}(x) \tan \left(\frac{\pi \alpha}{2}\right)\right]\right\}, & \alpha>1 \\ \exp \left\{\mathrm{i} \delta x-|c x|\left[1+\frac{2 \mathrm{i}}{\pi} \beta \operatorname{sign}(x) \ln x\right]\right\}, & \alpha=1\end{cases}
$$

Where $\mathrm{i}=\sqrt{-1}$, if $x \geq 0, \operatorname{sign}(x)=1$, otherwise, $\operatorname{sign}(x)=-1$. So et.al. [21] proposed a Fourier-series method to estimate the pdf and cdf based on its characteristic functions. Moreover, $X \sim S_{\alpha, \beta, c, \delta}$ is a stable process with parameters $\alpha, \beta, c, \delta$.

To investigate the heteroskedasticity of returns on market index, Mittnik et.al. [22] developed the stable Paretian power-GARCH model, they discussed the necessary and sufficient conditions for the GARCH model with stable innovation distributions. In this paper, we employed the stable-GARCH model and included the GJR frameworks to describe the asymmetric impact of good news and bad news. The model is as follows,

$$
\begin{aligned}
& y_{t}=a_{0}+\sum_{i=1}^{m} a_{i} y_{t-i}+c_{t} \epsilon_{t}, \epsilon_{t} \sim S_{\alpha, \beta, 1,0} \\
& c_{t}^{\alpha}=b_{0}+\sum_{i=1}^{p} b_{1 i}\left|\epsilon_{t-i}\right|^{\alpha}+\sum_{i=1}^{p} b_{1 i}^{-} I\left(\epsilon_{t-i}\right)\left|\epsilon_{t-i}\right|^{\alpha}+\sum_{j=1}^{q} b_{2 j} c_{t-j}^{\alpha}+\epsilon_{t},
\end{aligned}
$$

Where $I(x)=1$, if $x<0$, otherwise, $I(x)=0$. To address the autocorrelation on $y_{t}$, we applied the Ljung-Box-Q test to find the optimal number of lags on Eq. (2). The residuals on Eq. (2) followed the standard stable Paretian distributions. $c_{t}$ are the time-varying scale factors and followed GJR-GARCH model. Since the second or higher moments in stable Paretian distribution are infinite when $\alpha<2$, the exponent in GJR-GARCH terms will be replaced by $\alpha$ as introduced in [22]. This model will reduce to the normal GJR-GARCH model when $\alpha=2$. Moreover, the stable Paretian distributions had many advantages over other fat-tailed distributions. The generalized central limit theorem can be applied to stable Paretian distributions and the sum of i.i.d. stable random variables is still a stable random variable. These properties are important on statistics inference and risk management.

\section{Data and Empirical Results}

The S\&P 500 daily index from $1^{\text {st }}$ January 2006 to $31^{\text {st }}$ December 2007 are collected from Federal Reserve Economic Data (FRED). The log changes of S\&P 500 index will be used as market returns $\left(y_{t}\right)$ on Eq. (2). There are 502 observational values in the sample period. According to the Ljung-Box-Q test, 1 lag is required to mitigate the autocorrelation on $y_{t}$. We used Nolan's algorithm [17] to run the regression on Eq. (2). This algorithm allowed the residuals followed the stable Paretian distributions. The estimates of parameters are $a_{0}=0.00012, a_{1}=-0.09302$. We compared the performance between stable GJR-GARCH $(1,1)$ model and normal GJR-GARCH $(1,1)$ model. The results are given in Table 1.

The number of observations in the sample is 502. The columns "std" and "p-value" are the standard deviations and the probability values of the parameters. $*$ and $* *$ are $95 \%$ and $99 \%$ significance levels We tested $b_{0} \neq 0, b_{i j}>0, \alpha<2$ and $\beta \neq 0$ in the hypothesis. LLF are the likelihood function values and LLR are the likelihood ratio -2 (LLF from stable - LLF from normal). The p-value of LLR is obtained by using chi-square distribution with 2 degrees of freedom.

Table 1 shows that the characteristic parameter in stable GJR-GARCH model is significantly less than 2 and the skewness is different from 0. Moreover, the likelihood ratio test also supported that the stable GJR-GARCH $(1,1)$ model performs better than Gaussian distribution. The results show that 
conditional distributions of market returns are asymmetric and have tail fatter than normal especially in 2006 and 2007. For the GJR-GARCH model, we find that good news $\left(\epsilon_{t} \geq 0\right)$ and bad news $\left(\epsilon_{t}<0\right)$ have asymmetric impact on market fluctuations. $b_{11}^{-}$is significantly greater than 0 on both stable and normal GJR-GARCH models while $b_{11}$ is insignificant. The results show that the bad news had higher influence on market fluctuations.

Table 1. Parameters estimates of stable GJR-GARCH(1,1) and normal GJR-GARCH(1,1) models

\begin{tabular}{c|l|l|l|l|l|l}
\hline & \multicolumn{3}{|l|}{ Stable GJR-GARCH(1,1) } & \multicolumn{3}{l}{ Normal GJR-GARCH(1,1) } \\
\hline $\mathbf{N = 5 0 2}$ & Parameters & std & p-value & Parameters & std & p-value \\
\hline $\boldsymbol{b}_{\mathbf{0}}$ & $1.01 \mathrm{E}-06$ & $4.73 \mathrm{E}-07$ & $0.0332^{*}$ & $1.17 \mathrm{E}-06$ & $4.09 \mathrm{E}-07$ & $0.0044^{* *}$ \\
\hline $\boldsymbol{b}_{\mathbf{1 1}}$ & 0.0010 & 0.0487 & 0.4918 & 0.0010 & 0.0297 & 0.4865 \\
\hline $\boldsymbol{b}_{\mathbf{1 1}}^{-}$ & 0.0584 & 0.0283 & $0.0120^{*}$ & 0.0655 & 0.0340 & $0.0273^{*}$ \\
\hline $\boldsymbol{b}_{\mathbf{2 1}}$ & 0.9214 & 0.0534 & $<0.0001^{* *}$ & 0.8959 & 0.0366 & $<0.0001^{* *}$ \\
\hline $\boldsymbol{\alpha}$ & 1.8433 & 0.0144 & $<0.0001^{* *}$ & & & \\
\hline $\boldsymbol{\beta}$ & -0.9520 & 0.4429 & $0.0321^{*}$ & & & \\
\hline LLF & 1760.89 & & & 1733.79 & & \\
\hline LLR & 54.2107 & & $<0.0001^{* *}$ & & & \\
\hline
\end{tabular}

From the above data, we found that the Gaussian distribution may not be suitable for modeling the market returns especially for the crisis periods. The fat tail means the probability of extreme events is much higher than that in the normal distribution. The negative skewness also reveals that the chance of downside movement is higher than the upside movement on market index. In addition to the asymmetric impact of bad news to the market fluctuations, we can conclude that the market had higher downside risk than upside risk. Moreover, the downside risk has higher influence on the market fluctuation before the subprime mortgage crisis.

\section{Conclusion}

This paper investigated the distribution of returns on market index. We applied the stable family to release the distribution in GARCH models. The stable Paretian distribution had four parameters. They captured the thickness of the tail $(\alpha)$, the skewness $(\beta)$, the scale $(c)$ and the location $(\delta)$. Moreover, stable Paretian distribution nested the normal distribution. In other words, the normal distribution is one of members of the stable family. The generalized central limit theorem and the stable distribution are close under summation. We used the daily returns of S\&P 500 index as the market returns in our models, and we first addressed the autocorrelation by using the Nolan's algorithm. This algorithm allowed the distribution of the residuals followed stable Paretian distribution. To address the heteroskedasticity, we used stable GJR-GARCH model. The empirical results showed that conditional distribution under the GJR-GARCH model was still asymmetric and had tail fatter than normal. Moreover, we found that the bad news had higher impact on the market fluctuations rather than good news. These results provided some evidences that using the Gaussian distribution on risk management may not be suitable to evaluate the extreme losses of market. It may be an important reason that the damage from the subprime mortgage crisis is much higher than we expected.

\section{Acknowledgement}

This research was financially supported by the Macao Foundation and the Faculty Research Grant, No.: FRG-18-008-MSB.

\section{References}

[1] F. Lamantia, S. Ortobelli and S. Rachev, "An Empirical Comparison among VaR Models and 
Time Rules with Elliptical and Stable Distributed Returns," Investment Management and Financial Innovations, vol. 3, pp. 8-29, 2006.

[2] T. Bollerslev, "Generalized Autoregressive Conditional Heteroskedasticity," Journal of Econometrics, vol. 31, pp. 307-327, 1986.

[3] E. Fama, "Mandelbrot and the Stable Paretian Hypothesis," Journal of Business, vol. 36, pp. 420-429, 1963.

[4] B. Mandelbrot, "The Variation of Certain Speculative Prices," Journal of Business, vol. 36, pp. 394-419, 1963.

[5] J. H. McCulloch, "Measuring Tail Thickness to Estimate the Stable Index $\alpha$ : A Critique," Journal of Business \& Economic Statistics, vol. 15, pp. 74-81, 1997.

[6] F. X. Diebold, T. Schuermann and J. D. Stroughair, "Pitfalls and Opportunities in the Use of Extreme Value Theory in Risk Management," Journal of Risk Finance, vol. 1, pp. 30-35, 2000.

[7] T. G. Bali, "A Generalized Extreme Value Approach to Financial Risk Measurement.," Journal of Money, Credit and Banking, pp. 1613-1649, 2007.

[8] R. F. Engle, "Autoregressive Conditional Heteroscedasticity with Estimates of the Variance of United Kingdom Inflation," Econometrica, vol. 50, pp. 987-1007, 1982.

[9] T. Bollerslev, "A Conditional Heteroskedasticity Time Series Model for Speculative Prices and Rates of Return," Review of Economics and Statistics, vol. 69, pp. 542-557, 1987.

[10] V. Acharya, R. F. Engle and M. Richardson, "Capital Shortfall: A New Approach to Ranking and Regulating Systemic Risks," American Economic Review, vol. 102, pp. 59-64, 2012.

[11] T. Adrian and M. K. Brunnermeier, "CoVar," 2011.

[12] E. Fama, "Portfolio Analysis in a Stable Paretian Market," Management Science, vol. 11, pp. 404-419, 1965.

[13] E. Fama, "The Behavior of Stock Market Prices," Journal of Business, vol. 38, pp. 34-105, 1965.

[14] F. Allen and D. Gale, "Bubbles and Crises," Economic Journal, vol. 110, pp. 236-255, 2001.

[15] J. H. McCulloch, "Continuous Time Processes with Stable Increments," The Journal of Business, vol. 51, pp. 601-619, 1978.

[16] J. P. Nolan, "Maximum Likelihood Estimation and Diagnostics for Stable Distributions," 2001.

[17] J. P. Nolan and D. Ojeda-Revah, "Linear and nonlinear regression with stable errors," Journal of Econometrics, vol. 172, pp. 186-194, 2013.

[18] R. F. Engle and V. K. Ng, "Measuring and Testing the Impact of News on Volatility," Journal of Finance, vol. 48, pp. 1749-1778, 1993.

[19] V. M. Zolotarev, One-Dimensional Stable Distributions, American Mathematical Society, Providence, RI, 1986.

[20] J. P. Nolan, Stable Distributions - Models for Heavy Tailed Data, Boston: Birkhauser, 2015.

[21] J. C. So, U. S. C., D. Ding and L. Liu, "An Efficient Fourier Expansion Method for Calculation of Value-at-Risk: Contributions of Extra Ordinary Risks," International Journal of Financial Engineering, Preprint, 2015.

[22] S. Mittnik, M. S. Paolella and S. T. Rachev, "Stationarity of stable power-GARCH processes," Journal of Econometrics, vol. 106, pp. 97-107, 2002. 